\title{
Annotation of Siberian larch genome draft assembly
}

Bondar E.I. ${ }^{1,2}$, Feranchuk S.I. ${ }^{1}$, Birukhov V.V. ${ }^{1,2}$, Kuzmin D.A. ${ }^{1,3}$, Sharov V.V. ${ }^{1,2,3}$, Oreshkova N.V.., 2, 4 , Krutovsky K.V.1, 5, 6,7

${ }^{1}$ Laboratory of Forest Genomics, Institute of Fundamental Biology and Biotechnology, Siberian Federal University, Krasnoyarsk, Russia

${ }^{2}$ Laboratory of Genomic Research and Biotechnology, Federal Research Center "Krasnoyarsk Science Center," Siberian Branch, Russian Academy of Sciences, Krasnoyarsk, Russia

${ }^{3}$ Department of High Performance Computing, Institute of Space and Information Technologies, Siberian Federal University, Krasnoyarsk, Russia

${ }^{4}$ Laboratory of Forest Genetics and Selection, V. N. Sukachev Institute of Forest, Siberian Branch, Russian Academy of Sciences, Krasnoyarsk, Russia

${ }^{5}$ Department of Forest Genetics and Forest Tree Breeding, Georg-August University of Göttingen, Göttingen, Germany

${ }^{6}$ Laboratory of Population Genetics, N.I. Vavilov Institute of General Genetics, Russian Academy of Sciences, Moscow, Russia

${ }^{7}$ Department of Genomics and Bioinformatics, Institute of Fundamental Biology and Biotechnology, Siberian Federal University, Krasnoyarsk, Russia

*email: bone-post@ya.ru

The main objective of this work was to provide a verified and reliable annotation for the draft genome assembly of Siberian larch, a conifer tree that is known for its frosthardiness, fast growth, and rot-resistant high-quality timber. The MAKER2 annotation pipeline was used for automated gene annotation. The transcriptome assemblies from five tissue types assembled using the TrinityRnaSeq package were used as speciesspecific RNA-seq evidence. RepeatMasker with custom de novo repeat library generated by RepeatModeler was used for masking repeated genomic regions. Gene prediction was done using AUGUSTUS, which was iteratively trained on the verified set of annotated transcripts, preliminarily assembled with TopHat and Cufflinks. Functional annotation was performed using Blast2GO within the OmixBox Platform. Repeating elements comprised $4.8 \mathrm{Gbp}$ or $\sim 39 \%$ of the $12 \mathrm{Gbp}$ genome. In total, 50,163 gene models were obtained consisting of 151,838 exons and 101,675 introns. Repeat content in the intron sequences was lower than in the entire genome, but $12.9 \%$ of introns included transposable elements. GO category assignment based on InterProScan domains identification and BLAST homology search yielded 39253 gene models (77\%) with at least one assigned GO term. 